\title{
LEVELS OF RESISTIN AND VISFATIN IN PATIENTS WITH METABOLIC SYNDROME
}

\author{
Ginka H. Rayanova, \\ Silvia S. Ganeva, \\ Katya N.Todorova, \\ Tsvetan H. Lukanov', \\ Svetla P. Gecheva ${ }^{1}$
}

\author{
Clinic of Endocrinology and \\ Metabolic Diseases \\ ${ }^{1}$ Medico-Diagnostic Laboratory of \\ Immunology \\ Medical University - Pleven
}

\author{
Corresponding Author: \\ Ginka H. Rayanova \\ Clinic of Endocrinology and Metabolic \\ Diseases \\ University Hospital - Pleven \\ Pleven, 5800 \\ Bulgaria \\ e-mail: grayanova@abv.bg
}

Received: March 18, 2015

Revision received: March 22, 2015

Accepted: May 18, 2015

\begin{abstract}
Summary
The aim of the study is to investigate the serum levels of adipokines-resistin and visfatin in patients with metabolic syndrome. A prospective study was performed, including 153 (103 female, 50 male) subjects with metabolic syndrome. Carbohydrate metabolism was estimated by oral glucose tolerance test with $75 \mathrm{~g}$ of glucose. Of the subjects investigated, 40 were with normal glycaemic tolerance, 40 - with impaired fasting glycaemia, 28 - with impaired glucose tolerance and 45- with newly diagnosed diabetes mellitus type 2 . The results were compared to those in a control group of 20 subjects without findings indicating metabolic syndrome. The serum levels of resistin and visfatin were measured with an enzyme immunoassay method (ELISA, BioVendor Laboratory Medicine, Inc., Czech Republic). There were significant differences in body mass index, waist circumference, systolic blood pressure, plasma level of blood glucose and serum level of triglycerides between the examined subjects with metabolic syndrome, as compared with the control group. The amount of serum resistin in subjects with metabolic syndrome and newly diagnosed diabetes mellitus type 2 was significantly higher, as compared to the controls. Subjects with metabolic syndrome and pathological glucose tolerance- impaired fasting glycaemia, impaired glucose tolerance and newly diagnosed diabetes mellitus type 2 were found with a significantly higher serum level of visfatin, as compared to normal glucose tolerance and to the controls. Subjects with metabolic syndrome and pathological glucose tolerance- impaired fasting glucose, impaired glucose tolerance and newly diagnosed diabetes mellitus type 2 exhibited significantly changes in serum levels of adipokines- resistin and visfatin.
\end{abstract}

Key words: metabolic syndrome, fat tissue, adipokines, resistin, visfatin

\section{Introduction}

The ongoing global epidemic of diabetes mellitus type 2 is propelled by a concurrent rise in the prevalence of visceral obesity, insulin resistance and metabolic syndrome (MS). Adipose tissue is a known endocrine organ secreting several soluble factors, known as adipocytokines or adipokines. In normal healthy adults, adipocytokines regulate utilization and storage of lipids and help coordinate 
their distribution throughout the body. Their actions can be endocrine, paracrine, and autocrine as well. Many of these adipocytokines have a physiological role in metabolic functions and glucose metabolism [1, 2]. Adiponectin, leptin, resistin and, more recently, visfatin are adipocytokines that can partly explain the link between obesity, insulin resistance, metabolic syndrome, beta-cell dysfunction, endothelial dysfunction, and atherosclerosis [3, 4]. Resistin is a member of a class of cysteine-rich proteins collectively termed resistin-like molecules. It is secreted by human adipocytes and expressed by the macrophages embedded in fat tissue. This recently discovered fat tissue hormone is thought to represent a link between obesity and diabetes mellitus type 2 [5]. Data on levels of circulating resistin in patients with metabolic syndrome are very inconsistent. Some researchers found the resistin levels in obese patients to be significantly higher than those with normal-weight patients, while others found no significant differences in obesity and metabolic syndrome [6,7]. Visfatin is an adipokine identified in 2004 and thus named because of the suggestion that it would be predominantly produced and secreted in visceral fat. Fukuhara et al. have described visfatin as an adipokine that lowers plasma glucose and is able to bind to the insulin receptor and activate it by triggering the tyrosine phosphrylation cascade [8]. It stimulates the glucose uptake by adipocytes and myocytes and suppresses glucose release from liver cells; and is involved in the formation of adipocytes. Visfatin acts as an insulin mimetic despite the fact that it circulates in much lower concentrations than those of insulin [9].

The purpose of this research is to assess the serum levels of adipokines- resistin and visfatin in patients with metabolic syndrome.

\section{Material and Methods}

The research covered 153 individuals (103 women and 50 men) with metabolic syndrome (MS). MS is diagnosed if 3 of the specified 5 criteria of the International Diabetic Federation are available (diagnostic criteria for MS, IDF, 2010) [10] Table 1. The results were compared with a control group of 20 subjects not meeting the criteria for MS. Informed consent was obtained from all the subjects studied and the controls. Anthropometric methods were applied height and body weight measuring, determination of the body mass index (BMI). The waist circumference $(\mathrm{cm})$ was measured on a horizontal plain located in the middle between the lower edge of the 12th rib and the upper edge of the iliac bone, with accuracy up to $0.5 \mathrm{~cm}$; the average value of two measurements was recorded. The arterial pressure $(\mathrm{mm} \mathrm{Hg}$ ) was measured in a sitting position under standard conditions, after a 5-minute rest, at an interval of 5 minutes between two successive measurements. It was assumed that arterial hypertension was present at values $\geqslant 130 \mathrm{~mm}$ $\mathrm{Hg}$ for the systolic and/ or $\geqslant 85 \mathrm{~mm} \mathrm{Hg}$ for the diastolic arterial pressure or if patients with a history of arterial hypertension were taking antihypertensive drugs. Carbohydrate metabolism was assessed by conducting oral glucose tolerance test (OGTT) with $75 \mathrm{~g}$ glucose. Blood glucose was measured in venous plasma at the 0,60 th and 120th minute by applying the glucose-oxidase method (Glucose Analyzer Beckman). The lipid profile was assessed (general cholesterol, HDL-cholesterol, LDLcholesterol, triglycerides) in a fasting state. The general cholesterol, HDL-cholesterol and triglycerides were assessed by the enzymecolorimetric method (GPO- PAP; Biocon ${ }^{\circledR}$ Diagnostik), LDL-cholesterol was calculated by the Friedwald formula (LDL-cholesterol = general cholesterol - HDL-cholesterol triglycerides/2.2). The serum levels of resistin and visfatin were measured with an enzyme immunoassay method (ELISA, BioVendor Laboratory Medicine, Inc., Czech Republic).

Data analysis and processing was made with a statistical package for biostatical analysis SPSS version 17.0 (SPSS Chicago, USA). The indices studied are presented as average values $\pm \mathrm{SD}$ or as ratios; $p<0.05$ was adopted as level of statistical significance for all analyses.

Table 1. Diagnostic criteria for metabolic syndrome

\begin{tabular}{lll}
\hline Diagnostic criteria & Men & Women \\
\hline Waist circumference $(\mathrm{cm})$ & $>94$ & $>80$ \\
\hline Triglycerides $(\mathrm{mmol} / \mathrm{l})$ & $\geqslant 1.7$ & $\geqslant 1.7$ \\
\hline HDL-cholesterol $(\mathrm{mmol} / \mathrm{l})$ & $<1.03$ & $<1.29$ \\
\hline Blood pressure $(\mathrm{mmHg})$ & $\geqslant 130 / 85$ & $\geqslant 130 / 85$ \\
\hline Fasting blood glucose $(\mathrm{mmol} / \mathrm{l})$ & $\geqslant 5.6$ & $\geqslant 5.6$ \\
\hline
\end{tabular}




\section{Results}

The clinical characteristics of the patients studied and the control group are shown on Table 2. All individuals with MS included in the research met at least 3 of the specified criteria of the International Diabetes Federation (IDF) of 2010. According to the glucose tolerance, the patients were distributed in the following two groups: group one - individuals with normal glucose tolerance (normal GT; $n_{1}=40$ ) with fasting blood glucose $<5.6 \mathrm{mmol} / 1$ and group two - individuals with pathological glucose tolerance (pathological GT; $\mathrm{n}_{2}=113$ ). The patients of the second group were distributed in three subgroups: subgroup $1\left(\mathrm{n}_{2 \text { subgr. }}=40\right)$ individuals with impaired fasting glycaemia (IFG), subgroup $2\left(\mathrm{n}_{2}\right.$ subgr.2 $=28$ ) individuals with impaired glucose tolerance (IGT) and subgroup $3\left(\mathrm{n}_{2}\right.$ subgr.3 $\left.=45\right)$ individuals with newly diagnosed type 2 diabetes mellitus (NDMt2). Individuals with fasting blood glucose $\geqslant 5.6 \mathrm{mmol} / 1$ and $<7.0 \mathrm{mmol} / 1$ were included in the group of IFG [11]. Table 3 shows the clinical characteristics of the individuals in the groups - normal glucose tolerance, impaired fasting glycaemia, impaired glucose tolerance and newly diagnosed type 2 diabetes mellitus. The results show normal GT in $26.20 \%$ of patients, in much higher number of patients i.e. $73.80 \%$ carbohydrate metabolism disorders were identified; $(\mathrm{P}<0.05)$. The largest group was that of individuals with NDMt $2-45$ (29.40\%), followed by the individuals with IFG -
$40(26.10 \%)$, the lowest number of patients had IGT $-28(18.30 \%)$. The average age of the individuals included in the research was 42.65 \pm 13.0 years, and it was similar to the average age of the controls $-43.26 \pm 12.0$ years. Patients with pathological glucose tolerance were older than those with normal glucose tolerance and the age of the individuals with NDMt 2 was much higher than that of the patients with IFG and IGT; ( $p$ $<0.05)$. There were significant differences regarding body mass index, waist circumference, systolic blood pressure, plasma level of blood glucose, serum level of triglycerides between the subjects with MS studied and the controls. The average body mass index (BMI) of the studied patients was $35.52 \pm 7.2 \mathrm{~kg} / \mathrm{m}^{2}$. Individuals with pathological glucose tolerance had BMIs similar to those with normal glucose tolerance. Considerable difference in the waist circumference was proven between the individual groups, and waist circumference was highest in the three groups with disorders in glucose tolerance. The amount of serum resistin (ng/ml) in subjects with MS and NDMt2 was significantly higher $(1.85 \pm 0.7)$ as compared to the control group $(0.71 \pm 0.31) ;(\mathrm{P}=0.04)$. Subjects with IFG group showed non-significantly higher level of resistin as compared to normal GT and IGT (Table 4). Subjects with MS and pathological glucose tolerance (IFG, IGT, NDMt2) showed a significantly higher serum level of visfatin $(\mathrm{ng} / \mathrm{ml})$ as compared to normal GT and to the control group (Table 4).

Table 2. Clinical characteristics of investigated patients with metabolic syndrome and control group

\begin{tabular}{llll}
\hline Parameter & Control group & Patients with MS & P \\
\hline Number of patients & 20 & 153 & \\
\hline Mean age (years) & $43.26 \pm 12.00$ & $42.65 \pm 13.00$ & $\mathrm{NS}$ \\
\hline Body mass index $\left(\mathrm{kg} / \mathrm{m}^{2}\right)$ & $21.64 \pm 2.33$ & $35.52 \pm 7.20$ & $\mathrm{P}<0.05$ \\
\hline Waist circumference $(\mathrm{cm})$ & $76.42 \pm 3.56$ & $112.42 \pm 15.02$ & $\mathrm{P}<0.05$ \\
\hline Systolic blood pressure $(\mathrm{mmHg})$ & $116.32 \pm 7.61$ & $132.32 \pm 17.62$ & $\mathrm{P}<0.05$ \\
\hline Diastolic blood pressure (mmHg) & $77.37 \pm 6.42$ & $85.01 \pm 9.41$ & $\mathrm{NS}$ \\
\hline Fasting blood glucose $(\mathrm{mmol} / \mathrm{l})$ & $4.89 \pm 0.45$ & $6.47 \pm 1.85$ & $\mathrm{P}<0.05$ \\
\hline Total cholesterol $(\mathrm{mmol} / \mathrm{l}$ & $5.44 \pm 1.2$ & $5.21 \pm 1.09$ & $\mathrm{NS}$ \\
\hline HDL-cholesterol (mmol/l) & $1.45 \pm 0.39$ & $1.97 \pm 0.20$ & $\mathrm{NS}$ \\
\hline LDL-cholesterol $(\mathrm{mmol} / \mathrm{l})$ & $3.47 \pm 1.05$ & $3.31 \pm 1.39$ & $\mathrm{NS}$ \\
\hline Triglycerides $(\mathrm{mmol} / \mathrm{l})$ & $1.00 \pm 0.15$ & $1.96 \pm 0.16$ & $\mathrm{P}<0.05$ \\
\hline
\end{tabular}


Table 3. Clinical characteristics of patients with metabolic syndrome according to glucose tolerance groups

\begin{tabular}{lllll}
\hline Parameter & $\begin{array}{l}\text { Normal GT } \\
(\mathbf{n = 4 0})\end{array}$ & $\begin{array}{l}\text { IFG } \\
(\mathbf{n = 4 0})\end{array}$ & $\begin{array}{l}\text { IGT } \\
(\mathbf{n = 2 8})\end{array}$ & $\begin{array}{l}\text { NDMt2 } \\
(\mathbf{n}=\mathbf{4 5})\end{array}$ \\
\hline Mean age (years) & $32.83 \pm 8.74$ & $44.93 \pm 13.34$ & $43.11 \pm 10.91$ & $49.22 \pm 12.3$ \\
\hline Body mass index $\left(\mathrm{kg} / \mathrm{m}^{2}\right)$ & $33.33 \pm 6.73$ & $34.03 \pm 5.92$ & $37.10 \pm 9.00$ & $34.83 \pm 5.92$ \\
\hline Waist circumference (cm) & $109.65 \pm 14.89$ & $112.95 \pm 19.28$ & $113.39 \pm 19.28$ & $112.64 \pm 12.92$ \\
\hline Systolic blood pressure (mmHg) & $126.88 \pm 16.71$ & $137.13 \pm 19.58$ & $135.0 \pm 8.05$ & $131.22 \pm 15.23$ \\
\hline $\begin{array}{l}\text { Diastolic blood pressure } \\
\text { (mmHg) }\end{array}$ & $84.0 \pm 9.62$ & $85.62 \pm 7.94$ & $87.86 \pm 9.07$ & $83.56 \pm 10.42$ \\
\hline Fasting blood glucose (mmol/1) & $4.80 \pm 0.49$ & $6.18 \pm 0.40$ & $5.50 \pm 0.73$ & $8.82 \pm 1.50$ \\
\hline Total cholesterol (mmol/1 & $4.90 \pm 0.93$ & $5.30 \pm 1.09$ & $5.25 \pm 1.06$ & $5.40 \pm 1.21$ \\
\hline HDL-cholesterol (mmol/l) & $1.02 \pm 0.17$ & $1.03 \pm 0.40$ & $1.36 \pm 0.66$ & $1.06 \pm 0.35$ \\
\hline LDL-cholesterol (mmol/l) & $3.32 \pm 0.79$ & $3.44 \pm 0.99$ & $3.26 \pm 1.01$ & $3.21 \pm 1.01$ \\
\hline Triglycerides (mmol/l) & $1.44 \pm 0.30$ & $1.76 \pm 0.40$ & $2.12 \pm 0.40$ & $2.52 \pm 0.40$ \\
\hline
\end{tabular}

Table 4. Level of resistin in control group and in patients with metabolic syndrome according to glucose tolerance groups

\begin{tabular}{llllll}
\hline Parameter & $\begin{array}{l}\text { Gontrols } \\
(\mathbf{n = 2 0})\end{array}$ & $\begin{array}{l}\text { Normal GT } \\
(\mathbf{n = 4 0 )}\end{array}$ & $\begin{array}{l}\text { IFG } \\
(\mathbf{n = 4 0})\end{array}$ & $\begin{array}{l}\text { IGT } \\
(\mathbf{n = 2 8})\end{array}$ & $\begin{array}{l}\text { NDMt2 } \\
(\mathbf{n}=\mathbf{4 5})\end{array}$ \\
\hline Resistin (ng/ml) & $0.71 \pm 0.31$ & $0.86 \pm 0.21$ & $1.06 \pm 0.26$ & $0.93 \pm 0.15$ & $1.85 \pm 0.70^{*}$ \\
\hline Visfatin $(\mathrm{ng} / \mathrm{ml})$ & $0.35 \pm 0.11$ & $0.40 \pm 0.12$ & $0.55 \pm 0.10^{\S}$ & $0.61 \pm 0.20^{\S}$ & $0.90 \pm 0.21^{\S}$ \\
\hline
\end{tabular}

* indicates significant difference between NDMt2 and control group; ${ }^{\S}$ - vs. control and vs. normal GT ( $\left.<<0.05\right)$

\section{Discussion}

The results from this study conducted on patients with metabolic syndrome showed high prevalence of pathological glucose tolerance $73.80 \%$. The prevalence of the newly diagnosed type 2 diabetes mellitus in the studied group with MS was $29.4 \%$, that of IFG $-26.1 \%$ and that of IGT $-18.3 \%$, which is in conformity with the literature data demonstrating a five-fold higher risk of type 2 diabetes mellitus in individuals with the metabolic syndrome [12]. Our results confirmed the data from the study conducted among a random Bulgarian population in 2007. The authors found disorders of the carbohydrate metabolism in $73.9 \%$ of the participants who presented with the criteria for an MS. A newly diagnosed type 2 diabetes mellitus was present in $22.8 \%$ of these subjects, and impaired glucose tolerance - in $22.7 \%$. The IDF 2005 criteria were applied for diagnosis of the MS [13]. The prevalence of pathological glucose tolerance increases with age, and the individuals with newly diagnosed type 2 diabetes mellitus are considerably older than those with normal GT, IFG and IGT (Table 3). Similar data are found in other studies including patients with MS $[12,14]$. The most probable reasons are the reduction of insulin secretion and increasing of insulin resistance with age. The data in the literature about circulating serum level of resistin were controversial. Some researchers found the resistin levels in obese patients to be significantly higher than in patients those with normal weight patients, while others found no significant 
differences [6, 7]. Some investigators have suggested that resistin is a hormone that links visceral obesity to diabetes mellitus type 2 [5]. Experiments in humans have shown no differences in resistin expression among normal, insulin-resistant, MS and type 2 diabetes mellitus samples [15]. However, genetic studies have demonstrated an association between resistin and insulin resistance and MS [16]. The results from the conducted research showed increased level of resisitin only in patients with MS and NDMt2. The data in a previous study of ours showed that patients with MS and NDMt2 had significantly higher insulin resistance, as compared to subjects with MS and normal GT, IFG or IGT [14]. They have observed that resistin has been shown to antagonize insulin action; resistin levels are increased in diet-induced obesity as well as in genetic models of obesity and insulin resistance. Furthermore, resistin gene expression is markedly down-regulated by treatment with antidiabetic drugs called thiazolidinediones that improve target-tissue sensitivity to insulin. [16, 17]. It has been found that human abdominal adipose tissue has a higher amount of resistin than other fat depots [18]. The results obtained in our study showed a significantly higher serum level of visfatin in subjects with MS and pathological glucose tolerance (IFG, IGT and NDMt2), as compared to subjects with normal glucose tolerance and the control group. The data in the literature about circulating serum level of visfatin in patients with MS were controversy. Some studies confirmed an increased level of circulating visfatin in obesity and MS [19]. However, results from other studies were contradictory in that they showed lower levels of serum visfatin in patients with MS [17]. Several studies have shown that plasma visfatin levels correlate highly with obesity, visceral fat mass, type 2 diabetes mellitus and metabolic syndrome. Visfatin concentrations are high in patients with metabolic syndrome, those that are overweight and obese, have greater waist circumference, high blood pressure, high fasting glucose levels, serum triglycerides and low HDL-cholesterol $[19,20]$.

\section{Conclusion}

Subjects with metabolic syndrome and pathological glucose tolerance- impaired fasting glucose, impaired glucose tolerance and newly diagnosed diabetes mellitus type 2 were found with significant changes in serum levels of resistin and visfatin. Physiological functions of adipkines have major roles in different metabolic processes and tissues. The adipokines- resistin and visfatin could serve as a missing link in the causal relationship between metabolic syndrome and comorbidities. The combination of the components of metabolic syndrome result in a twice higher risk of occurrence of cardiovascular diseases, they are associated with early atherosclerosis, impaired fibrinolysis, dyslipidemia, microalbuminuria and diseases such as polycystic ovary syndrome, nonalcoholic hepatic steatosis and non-alcoholic fatty liver disease (NAFLD), chronic obstructive lung disease, cancerogenesis, acanthosis nigricans and psoriasis vulgaris [21]. Resistin and visfatin may provide a biomarker for disease severity such as obesity, metabolic syndrome and diabetes mellitus type 2 [22]. Development of novel therapeutic procedures for obesity and obesity-associated diseases like metabolic syndrome and diabetes mellitus type 2 could possibly be achieved through an integral insight into resistin and visfatin, as well as insight into other adipokine functions.

\section{Acknowledgements}

Research project N4/2014, financed by Medical University, Pleven.

\section{References}

1. Galik S, Oakhill J, Steinberg G. Adipose tissue as an endocrine organ. Mol Cell Endocrinol. 2010;316(2):129-39.

2. Karastegiou K, Mohamed-Ali V. The autocrine and paracrine roles of adipokines. Mol Cell Endocrinol. 2010;318(1-2):69-78.

3. Koleva D, Orbetzova M, Atanasova P. Adipose tissue hormones and appetite and body weight regulators in insulin resistance. Folia Med. 2013;55(11):25-32.

4. Deng Y, Scherer P. Adipokines as novel biomarkers and regulators of the metabolic syndrome. Ann N Y Acad Sci. 2010;1212:E1-E19. doi: 10.1111/j.1749-6632.2010.05875.x.

5. Dogru T, Sonmez A, Tasci I, Bozoglu E. Yilmaz MI, Gene H. at al. Plasma resistin levels in patients with newly diagnosed untreated type 2 diabetes mellitus and impaired glucose tolerance. Diabetes Res Clin Pract. 2007;76 (1):2-7.

6. Norata GD, Ongari M, Garlaschelli K, Raselli S, Grigore L, Catapano AL. Plasma resistin levels 
correlate with determinants of the metabolic syndrome. Eur J Endocrinol. 2007;156(2):279-84.

7. Utzschneider K, Carr D, Tong J, Wallace T, Hull R, Zrika $\mathrm{S}$ at al. Resistin is not associated with insulin sensitivity or metabolic syndrome in humans. Diabetologia. 2005;48(11):2330-3.

8. Fukuhura A, Matsuda M, Nishizawa M. Visfatin: a protein secreted by visceral fat that mimics the effects of insulin. Science. 2005;5708(307):42630 .

9. Saddi-Rosa P, Olivera C, Giuffrida F, Reis A. Visfatin, glucose metabolism and vascular disease: a review of evidence. Diabetol Metab Syndr. 2010; 26;2:21. doi: 10.1186/1758-5996-221.

10. Christov Vl, Gocheva N, Petkova M, Zaharieva S, Tankova Tz, Orbetzova M, et al. A consensus of the Bulgarian Institute Metabolic Syndrome. Nauka Endocrinologia. 2010;2:3-70. (in Bulgarian)

11. World Health Organization. Definition and diagnosis of Diabetes Mellitus and intermediate Hyperglycaemia. Report of WHO/IDF Consultation. Geneva, World Health Org. 2006.

12. Tota-Maharaj R, Defilippis A, Blumenthal R, Blaha M. A practical approach to the metabolic syndrome: review of current concepts and management. Curr Opin Cardiol. 2010;25(5):50212.

13. Borissova A-M, Kovatcheva R, Shinkov A, Atanassova I, Vukov M, Aslanova $\mathrm{N}$ et al. Prevalence and features of the metabolic syndrome in unselected bulgarian population. Endocrinologia. 2007;2:68-77. (in Bulgarian)

14. Rayanova G, Ganeva S, Todorova K, Lukanov T, Gecheva S. Disorders of the carbohydrate metabolism in patients with metabolic syndrome. Endocrinologia. 2014;3:139-43. (in Bulgarian)
15. De Luis DA, Gonzalez SM, Conde R, Aller R, Izaola $\mathrm{O}$, Primo D. lack of association of serum resistin levels with metabolic syndrome criteria in obese patients. Clin Biochem. 2012;44(16):12803.

16. Silha V, Kresk M, Skrha J, Sucharda P, Nyomba B, Murphy L. Plasma resistin, adiponectin and leptin levels in lean and obese subjects: correlations with insulin resistance. Eur J Endocrinol. 2003;149(4):331-5.

17. Ebtesam DL-S and Shehzad A. Leptin, resistin and visfatin: the missing link between endocrine metabolic disorders and immunity. Eur J Med Res. 2013;18(1):12-29.

18. Rabe K, Lehrke M, Parhofer K, Broedi U. Adipokines and insulin resistance, Mol Med. 2008; 14 (11-12): 741-751.

19. Tan H, Zhong M, Gomg H,Wang S, Zhang Y, Zhang W. Increased serum visfatin in patients with metabolic syndrome. Clinical Endocrinology. 2008; 6(69):878-884.

20. Esteghamati A, Morteza A, Zandieh A, Jafari S, Rezaee M, Nakhjavani M. The value of visfatin in the prediction of metabolic syndrome: a multifactorial analysis. J Cardiovasc Tnansl Res. 2012;5(4):541-6.

21. Vasilescu R, Ifrim S, Trigoviste C. Relationship between plasma adipokines, inflammation, insulin resistance and subclinical atherosclerosis in newly diagnosed type 2 diabetes. Journal of Diabetes Mellitus. 2011;1(2):17-25.

22. Rasouli N, Kern P. Adipocytokines and metabolic complications of obesity. J Clin Endocrinol Metab. 2008;93(11):S64-S73. 